KING, D. J., Burke, M. \& LuCas, R. A. (1995) Antipsychotic drug-induced dysphoria. British Journal of Psychiatry. 167, $480-482$.

R. GRAY

C. BROWN

R. HOOGHAMER

A. LEE

Wonford House Hospital

S. OXBORROW

Exeter EX2 $5 A F$

AUTHOR'S REPLY: Gray et al make a number of criticisms which would, of course, be valid had ours been a prospective study. It was, however, submitted as a Brief Report and was an account of clinical observations made in the course of two pharmacokinetic studies. It was reported because it was probably the largest single series in healthy volunteers ( 51 subjects) to be published, and because of the high and consistent incidence of dysphoria (an unpleasant and subjective mood state characterised by irritability, hostility, fear, tension, panic, impatience or vaguely described distress) on the two study days $(40 \%)$.

The aims of the report were to remind clinicians not to miss or dismiss this symptom in their patients, to illustrate that dysphoria can occur independently from akathisia and to note that the "neuroleptic threshold" is unlikely to be any different in patients and volunteers after acute dosing (McEvoy et al, 1991).

McEvoy, J. P., Hogarty, G. E. \& Steingard, S. (1991) Optimal dose of neuroleptic in acute schizophrenia. A controlled study of the neuroleptic threshold and higher haloperidol dose. Archives of General Psychiatry. 48, 739-745.

The Queen's University of Belfast

D. J. KING

97 Lisburn Road

Belfast BT9 7BL

\section{Bias towards chronicity in schizophrenia?}

SIR: The conclusions of 13 years follow-up study by Mason et al (1995) raise important questions as much about the course of schizophrenia as to the 'bias' of the professionals. The conclusion "only $17 \%$... achieve complete recovery over the longer term" appears biased for the following reasons. It appears that mental health professionals are not willing to consider the illness of schizophrenia in a manner that general medicine considers illnesses like hypertension, diabetes, arthritis. The above three illnesses and many others with 'no cure' do not lead to 'complete recovery'. However, doctors working with non-psychiatric illnesses emphasise and focus on abilities rather than disabilities. The diabetic with food restriction among other limitations, the hypertensive with activity restriction, do not consider themselves as 'disabled' and learn to live near normal lives. All of them need help and care but they are not seen as 'Chronic' in the way we present the course of schizophrenia.

There are three implications for a revision of the 'non-recovery' approach to schizophrenia. Firstly, a medical approach to describe course would draw greater support for patients, research and professional work. Secondly, there is need to embark on a series of new studies with first episode patients to delineate how far chronicity is a function of events occurring after the onset of illness (early recognition, early treatment, adequate family and community support, rehabilitation in the community, lack of stigma etc.) rather than an essential part of the illness. Thirdly, for those of us working in developing countries, the difference in the approach of 'treatability' could prove decisive in terms of support from the planners and people for mental health programmes. This is especially true for the acceptance of the integration of mental health with primary health care which is the current focus of mental health care in developing countries. A shift in 'bias' of mental health professionals may be timely?

Mason, P., Harrison, G., Glazabrook, C., et al (1995) Characteristics of outcome in schizophrenia at 13 years. British Journal of Psychiatry, 167, 596-603.

National Institute of Mental Health

R. SRINIVASA MURTHY and Neurosciences

P.O. Box 2900

Bangalore-560029

India

AUTHORs' REPLY: We agree with Murthy's point that 'disability and chronicity' have been overemphasised in describing the outcome in schizophrenia, and that this pessimistic outlook is unlikely to be in the best interests of our patients. However, there are many authors who share a more optimistic view about the outcome of schizophrenia. Zubin et al (1983) suggested that the course of schizophrenia has undergone a 'benign metamorphosis' over the second half of this century, and Harding et al (1987) demonstrated good symptomatic outcome (no further signs or symptoms of schizophrenia) in $68 \%$ of their 20-25 year follow-up of 'chronic hopeless' cases of DSM-III schizophrenia. In a previous paper (Harrison \& Mason, 1993) we commented on the hypothesis that outcome in schizophrenia has undergone a 'benign metamorphosis'. We suggested 